\title{
ON EXTREME AVERAGING OPERATORS
}

\author{
S. P. LLOYD
}

1. Introduction. With $X$ a compact Hausdorff space, let $C(X)$ denote the Banach algebra of continuous scalar valued functions on $X$, with scalars either the real or the complex numbers, and with the usual pointwise operations and uniform norm. Let $a$ be a closed subalgebra of $C(X)$ containing constant functions, and complex conjugate functions in the complex case. We are concerned with the set $P(Q)$ of all positive bounded projections in $C(X)$ onto fixed range space $a$.

It has been shown by Birkhoff $[1]$ and others $[2 ; 3]$ that the members of $P(Q)$ are averaging operators, in the sense $\mu(f \mu g)=(\mu f)(\mu g)$, $f, g \in C(X)$, with structure of the following type. Subalgebras $Q$ of the kind specified correspond 1-1 to upper semicontinuous decompositions of $X$ into closed equivalence classes, the equivalence classes for a given $a$ consisting of the sets whose points are not separated by the functions in $a$. A given $\mu \in \mathcal{P}(a)$ determines for each equivalence class a regular Borel probability measure living on the equivalence class and for each $f \in C(X)$ the value of $\mu f$ on an equivalence class is obtained by averaging $f$ over the equivalence class with respect to the associated measure.

As it turns out, $P(Q)$ is a bounded convex set and we are interested in the extreme point properties of this convex set. Generalizing a result of Davis [4], we characterize the extreme points of $P(Q)$ as those positive projections which are algebraic homomorphisms of $C(X)$ onto $Q$. Examples are given where (i) $P(Q)$ is empty (ii) nonempty $P(Q)$ has no extreme points (iii) nonempty $P(Q)$ has a few extreme points but is larger than their convex hull (iv) nonempty $P(Q)$ is the closed convex hull of its extreme points. Situation (iv) obtains, in particular, when $X$ is the extremally disconnected Stone space of a finite measure algebra.

2. Preliminaries. Let $\phi$ denote the equivalence in $X$ associated with $a$. That is, $x_{1} \phi x_{2}$ if and only if $g\left(x_{1}\right)=g\left(x_{2}\right)$ for all $g \in a$. The quotient space $Y=X / \phi$ of equivalence classes is compact Hausdorff and the canonical continuous mapping $\pi: X \rightarrow Y$ is closed. The subalgebra $a$ is isometrically algebraically isomorphic to $C(Y)$ under the 1-1 correspondence $\rho: C(Y) \rightarrow a$ defined by

Presented to the Society, June 16, 1961 under the title Extreme means are homomorphisms; received by the editors October 9, 1961 and, in revised form, February 5, 1962. 


$$
\rho g(x)=g(\pi x),
$$

$x \in X, g \in C(Y)$.

We represent the conjugate space $C^{*}(X)$ of bounded linear functionals on $C(X)$ as the space of regular bounded scalar valued (signed) Borel measures on $X$, with total variation norm. We denote by $X$ the $\sigma$-field of Borel subsets of $X$. We denote by $(f, \lambda)$ the value $(f, \lambda)=\int f(x) \lambda(d x)$ of $\lambda \in C^{*}(X)$ at $f \in C(X)$.

Proof of the following representation theory for members of $P(Q)$ appears in $[1 ; 2 ; 3]$; we use the notation of Wright $[3]$.

TheOREM 1. The members of $P(Q)$ correspond 1-1 to certain $C^{*}(X)$ valued functions on $Y$, as follows. If $\sigma: Y \rightarrow C^{*}(X)$ corresponds to $\mu \in \mathcal{P}(\mathrm{Q})$ then $\sigma$ and $\mu$ are related by (i), and $\sigma$ has properties (ii)-(iv):

(i) $\mu f(x)=\left(f, \sigma_{\pi x}\right)=\int f\left(x^{\prime}\right) \sigma_{\pi x}\left(d x^{\prime}\right), x \in X, f \in C(X)$,

(ii) $\sigma: Y \rightarrow C^{*}(X)$ is continuous with the $C(X)$ (weak*) topology in $C^{*}(X)$,

(iii) $\sigma_{y} \geqq 0, \sigma_{y}(X)=1, y \in Y$,

(iv) $\sigma_{y}(E)=\sigma_{y}\left(E \cap \pi^{-1} y\right), E \in X, y \in Y$.

3. Extreme points. A cross section of the mapping $\pi: X \rightarrow Y$ is any continuous function $\gamma: Y \rightarrow X$ such that $\pi \gamma$ is the identity mapping on $Y$. When the measure $\sigma_{y}$ of (2) is concentrated at a single point $\gamma(y) \in \pi^{-1} y$ for each $y \in Y$ then $\gamma: Y \rightarrow X$ is a cross section of $\pi$, from (2.ii) and (2.iii). The corresponding projection is an algebraic homomorphism of $C(X)$ onto $a$; in fact, we have (as noted by Wright [3])

THEOREM 2. The members of $\mathcal{P}(\mathbb{Q})$ which are homomorphisms of $C(X)$ onto a correspond 1-1 to cross sections of $\pi: X \rightarrow Y$.

The connection with extreme points is given by

Theorem 3. A positive projection $\mu \in \mathcal{P}(Q)$ is an extreme point of $P(a)$ if and only if $\mu$ is an algebraic homomorphism of $C(X)$ onto $a$.

Proof. The if part of the statement is due to Wright [3]; we reproduce the proof for the sake of completeness. Suppose $\mu \in \mathcal{P}(Q)$ is a homomorphism. In representation (2), this becomes $\left(f g, \sigma_{y}\right)$ $=\left(f, \sigma_{y}\right)\left(g, \sigma_{y}\right), y \in Y, f, g \in C(X)$, which is to say that for each $y \in Y$ the functional $\sigma_{y} \in C^{*}(X)$ is a homomorphism of $C(X)$ into the scalars, and onto because (2.iii) implies $\left(1, \sigma_{y}\right)=1$. It is well known that $\sigma_{y}$ must then be for each $y$ a unit point measure, that is, an extreme point of the set of regular Borel probability measures on $X$. It follows at once that $\mu$ is necessarily an extreme point of $P(Q)$. Conversely, suppose $\mu$ is an extreme point of $P(Q)$. We will show that 
the corresponding $\sigma$ has the property that for each $y \in Y$ and $E \in X$, either $\sigma_{\nu}(E)=0$ or $\sigma_{\nu}(E)=1$. It will then follow from well-known arguments that $\sigma_{y}$ is for each $y$ a homomorphism of $C(X)$ onto the scalars, and hence $\mu$ a homomorphism onto $a$. Suppose to the contrary that $0<\sigma_{\nu_{0}}\left(E_{0}\right)<1$ obtains for some $y_{0} \in Y, E_{0} \in X$. Since $\sigma_{\nu_{0}}$ is a regular Borel probability measure, there exist closed sets $F_{1} \subset E_{0}$, $F_{2} \subset E_{0}^{\prime}$, such that $\sigma_{\nu_{0}}\left(F_{1}\right)>0, \sigma_{\nu_{0}}\left(F_{2}\right)>0$. Because $X$ is normal, there exists $f_{1} \in C(X)$ such that $f_{1}\left(F_{1}\right)=1, f_{1}\left(F_{2}\right)=0,0 \leqq f_{1} \leqq 1$. With $f_{2}=1-f_{1}$, define $\tau^{(i)}: Y \rightarrow C^{*}(X)$ by

$$
\tau_{y}^{(i)}(E)=\int_{E} f_{i}(x) \sigma_{y}(d x), \quad y \in Y, E \in X, i=1,2,
$$

noting that $\tau^{(1)}+\tau^{(2)}=\sigma$. The measure $\tau_{\nu}^{(i)}$ lives on $\pi^{-1} y$, clearly, and from $\left(f, \tau_{\nu}^{(i)}\right)=\left(f f_{i}, \sigma_{\nu}\right), y \in Y, f \in C(X)$ (Radon-Nikodym) it follows that each $\tau^{(i)}$ induces a positive bounded linear mapping of $C(X)$ into $C(Y)$.

In particular, the nonnegative real functions $\tau_{y}^{(i)}(X)=\left(1, \tau_{\nu}^{(i)}\right)$ $=\left(f_{i}, \sigma_{\nu}\right)$ are continuous on $Y$, and moreover, $\tau_{\nu_{0}}^{(i)}(X) \geqq \sigma_{\nu_{0}}\left(F_{i}\right)>0$, $i=1,2$. For an arbitrary $a>0$ there exists then an open neighborhood $V$ of $y_{0}$ such that

$$
\left|\tau_{y}^{(i)}(X)-\tau_{y_{0}}^{(i)}(X)\right|<a \tau_{y_{0}}^{(i)}(X), \quad i=1,2, y \in V .
$$

Because $Y$ is completely regular, there exists $h \in C(Y)$ such that $h\left(y_{0}\right)=1, h\left(V^{\prime}\right)=0,0 \leqq h \leqq 1$. With $b$ to be chosen presently, define

$$
\begin{aligned}
\sigma_{y}^{(i)}(E)=\left[\frac{b h(y)}{\tau_{\nu_{0}}^{(i)}(X)}\right] \tau_{\nu}^{(i)}(E)+\left[1-\frac{b h(y)}{\tau_{\nu_{0}}^{(i)}(X)} \tau_{y}^{(i)}(X)\right] \sigma_{y}(E), \\
y \in Y, E \in X, i=1,2 .
\end{aligned}
$$

Now with $0<b<(a+1)^{-1}$, the continuous coefficients in square brackets above are nonnegative real on $Y$, and it is readily verified that each $\sigma^{(i)}$ induces a positive projection in $C(X)$ onto $a$. A straightforward calculation gives

$$
\left(f_{1}, \sigma_{y_{0}}^{(1)}\right)-\left(f_{1}, \sigma_{\nu_{0}}^{(2)}\right)=b \frac{\left(f_{1}^{2}, \sigma_{\nu_{0}}\right)-\left(f_{1}, \sigma_{y_{0}}\right)^{2}}{\left(f_{1}, \sigma_{y_{0}}\right)\left(f_{2}, \sigma_{y_{0}}\right)},
$$

and since $f_{1} \neq$ const almost everywhere $\left[\sigma_{y_{0}}\right]$, the above difference does not vanish; that is, $\sigma^{(1)}$ and $\sigma^{(2)}$ correspond to distinct means. Finally, the identity $\sigma=\tau_{\nu_{0}}^{(1)}(X) \sigma^{(1)}+\tau_{\nu_{0}}^{(2)}(X) \sigma^{(2)}$ shows that $\sigma$ does not correspond to an extreme mean, contrary to hypothesis. Hence it must be the case that $\sigma$ takes values 0 and 1 only, implying that 
each $\sigma_{y}$ is a homomorphism. (Proof as in $[5$, p. 444]; we omit the details.)

COROLlaRy. Extreme points of $P(a)$ correspond 1-1 to cross sections of $\pi: X \rightarrow Y$.

4. Some examples. The author is indebted to E. A. Michael for Example 1 and for a suggestion which led to Examples 2 and 3.

ExAmple 1. $P(a)$ is empty. Let $X$ be the interval $[0,3]$ and let $a$ consist of the functions in $C(X)$ which are constant on the subinterval $[1,2]$. The equivalence classes consist of the interval $[1,2]$ and the remaining individual points of $X$. From Theorem $1, \mu \in \mathcal{P}(a)$ would have the form

$$
\mu f(x)=\int_{1}^{2} f\left(x^{\prime}\right) \sigma\left(d x^{\prime}\right), x \in[1,2] ; \mu f(x)=f(x), x \in X-[1,2] .
$$

For no measure $\sigma$ is $\mu f$ continuous if $f(1) \neq f(2)$, and it follows that $P(a)$ is empty.

EXAmple 2. Nonempty $\odot(a)$ has no extreme points. Let $X$ be the interval $[0,2]$ with endpoints identified, i.e., a circle, and let $a$ consist of the functions $g$ in $C(X)$ satisfying $g(x)=g(x+1), 0 \leqq x<1$. The equivalence classes are the pairs $\{x, x+1\}, 0 \leqq x<1$, and $Y$ may be represented as the interval $[0,1]$ with endpoints identified. Let $s(x)$, $0 \leqq x \leqq 2$, be any continuous function satisfying $0 \leqq s(x) \leqq 1,0 \leqq x \leqq 2$, and $s(x)+s(x+1)=1,0 \leqq x \leqq 1$. Then $\mu: C(X) \rightarrow Q$ defined by $\mu f(x)$ $=\mu f(x+1)=s(x) f(x)+s(x+1) f(x+1), 0 \leqq x \leqq 1$, is a positive bounded projection onto $a$, as is easily verified. It is not hard to see that the canonical projection $\pi: X \rightarrow Y$ in this example has no cross sections. It follows from Theorem 3 corollary that $P(a)$ has no extreme points.

The mapping $\pi$ is open in Example 2, where projections exist, and not open in Example 1, where projections do not exist, and Michael [6] has shown that sufficient conditions for $P(a)$ to be nonempty are that $\pi$ be open and $X$ be metric. Openness of $\pi$ is not necessary for the existence of projections, however, as the next example shows.

ExAmple 3. Nonempty $P(a)$ has no extreme points; $\pi$ is not open. Let $X$ be the interval $[0,2]$, but now without identifications. Let $a$ consist of the functions $g$ in $C(X)$ satisfying $g(x)=g(x+1), 0 \leqq x \leqq 1$. The equivalence classes are the pairs $\{x, x+1\}, 0<x<1$, and the triple $\{0,1,2\}$. Again, $Y$ is a circle; $\pi$ is no longer open. Projections onto $a$ exist and have the same form as in Example 2, except that $s$ must now also satisfy $s(0)=s(2)=0$. It is still the case that $\pi$ has no cross sections, whence $P(Q)$ has no extreme points. 
Example 4. Nonempty $P(a)$ has a few extreme points. Let $X$ be the interval $[-1,1]$ and let $a$ consist of the even functions in $C(X)$. The equivalence classes are $\{0\}$ and the pairs $\{x,-x\}, 0<x \leqq 1$. From Theorem 1, the members of $P(Q)$ have the form $\mu f(x)$ $=s(|x|) f(|x|)+[1-s(|x|)] f(-|x|), 0<|x| \leqq 1 ; \mu f(0)=f(0)$, where $s$ is any function continuous on $0<x \leqq 1$ satisfying $0 \leqq s(x) \leqq 1$, $0<x \leqq 1$ (e.g., $s(x)=\sin ^{2}(1 / x)$ ). It is easy to see that $\pi$ has exactly two cross sections; the corresponding extreme projections are $\mu f(x)$ $=f(|x|),-1 \leqq x \leqq 1$, and $\mu f(x)=f(-|x|),-1 \leqq x \leqq 1$. The convex hull of these is obviously much smaller than $P(Q)$.

ExAmple 5. Nonempty $\rho(Q)$ is the closed convex hull of its extreme points. In a number of applications [3], $X$ is the Stone space of a finite measure algebra, and in this event $P(a)$ is nonempty and compact in a certain locally convex topology and hence (KreinMilman) the closed convex hull of its extreme points. To be explicit let $(\Omega, \mathcal{F}, P)$ be a finite measure space, and let $L_{\infty}(\Omega, \mathcal{F}, P)$ be the Banach algebra of $P$ essentially bounded scalar valued functions on $\Omega$. Then $L_{\infty}(\Omega, \mathcal{F}, P)$ is isometrically algebraically isomorphic to $C(X)$, where the extremally disconnected compact Hausdorff space $X$ is the Stone space of the measure algebra $F$ modulo $P$ null sets. Let $\mathcal{G}$ be a given sub- $\sigma$-field of $\mathcal{F}$. Under the isomorphism of $L_{\infty}(\Omega, \mathcal{F}, P)$ and $C(X)$, the subalgebra $L_{\infty}(\Omega, \mathcal{G}, P)$ of $\mathcal{G}$ measurable elements of $L_{\infty}(\Omega, \mathcal{F}, P)$ is isomorphic to a closed self-adjoint subalgebra $a$ of $C(X)$ containing constants. A positive projection in $L_{\infty}(\Omega, \mathcal{F}, P)$ onto $L_{\infty}(\Omega, \mathcal{G}, P)$ (e.g., the conditional expectation operator with respect to $\mathcal{G}$ ) becomes a positive projection in $C(X)$ onto $a$. A proof that $P(Q)$ is compact in a suitable topology can be obtained as an easy modification of the proof of Theorem 8.4 of reference [3]; we omit the details.

EXAMPLE 6. Nonempty noncompact $\rho(a)$ is the closed convex hull of its extreme points. Let $X$ be the unit square $\{(x, y): 0 \leqq x$, $y \leqq 1\}$. Let $Q$ consist of the functions of $x$ only. From Theorem 1 , each $\mu \in P(a)$ has the form $\mu f(x, y)=\int_{0}^{1} f\left(x, y^{\prime}\right) \sigma_{x}\left(d y^{\prime}\right)$, where for each $0 \leqq x \leqq 1, \sigma_{x}$ is a regular Borel probability measure on the unit interval. The extreme projections are clearly $\mu f(x, y)=f(x, y(x)),(x, y)$ $\in X$, where $y(x)$ is any function continuous on and satisfying $0 \leqq y(x) \leqq 1$ on $0 \leqq x \leqq 1$. We will show that any given open weak operator neighborhood $N_{\mu}$ of any given $\mu \in \mathcal{P}(Q)$ contains a convex combination of extreme projections. Consider the projection $\bar{\mu}$ whose corresponding $\bar{\sigma}$ is given by

$$
\bar{\sigma}_{x}(y)=\frac{h}{1+h} y+\frac{1}{h} \int_{0}^{y}\left[\sigma_{x}\left(y^{\prime}+y^{\prime} h\right)-\sigma_{x}\left(y^{\prime}+y^{\prime} h-h\right)\right] d y^{\prime} ;
$$


we abbreviate $\sigma_{x}([0, y])$ to $\sigma_{x}(y)$. It is not hard to see that $\bar{\mu} \in N_{\mu}$ if $h>0$ is sufficiently small. The measures $\bar{\sigma}_{x}$ have densities satisfying

$$
\frac{h}{1+h} \leqq \frac{\partial \bar{\sigma}_{x}(y)}{\partial y} \leqq \frac{h}{1+h}+\frac{1}{h}, \quad 0 \leqq x \leqq 1 .
$$

For each $0<\alpha<1$ and each $0 \leqq x \leqq 1$ let $y_{\alpha}(x)$ denote the (unique) solution of $\bar{\sigma}_{x}\left(y_{\alpha}(x)\right)=\alpha$. The various properties of $\bar{\sigma}$ insure that $y_{\alpha}(x)$ is continuous on $0 \leqq x \leqq 1$ for each fixed $0<\alpha<1$. Define $\mu_{n} \in \odot(Q)$ by

$$
\mu_{n} f(x, y)=\frac{1}{2^{n}-1} \sum_{j=1}^{2^{n}-1} f\left(x, y_{j / 2^{n}}(x)\right), \quad(x, y) \in X .
$$

Riemann-Stieltjes integration theory shows that $\mu_{n} f(x, y)$ converges to $\bar{\mu} f(x, y)$ for each $0 \leqq x \leqq 1$ and it follows that $\mu_{n} \in N_{\mu}$ for large enough $n$. Thus the convex hull of the extreme projections is dense in $P(Q)$ in the weak operator topology. To see that $P(Q)$ is not compact in this topology, consider the sequence $\mu_{n}$ defined by $\mu_{n} f(x, y)$ $=f\left(x, y_{n}(x)\right)$, where $y_{n}(x)=\min (n x, 1)$. This is a Cauchy sequence in the weak operator topology which does not converge to a member of $P(Q)$; compactness fails because of incompleteness.

5. Acknowledgments. The author wishes to thank Professor Fred B. Wright for his interest, and in particular for useful comments on a preliminary version of the present paper. A conversation with Ernest Michael was very helpful.

\section{REFERENCES}

1. G. Birkhoff, Moyennes de fonctions bornees, Algèbre et Théorie des Nombres, Colloques Internationaux du Centre National de la Recherche Scientifique, no. 24, 143153, Centre National de la Recherche Scientifique, Paris, 1950.

2. J. L. Kelley, Averaging operators on $C_{\infty}(X)$, Illinois J. Math. 2 (1958), 214223.

3. F. B. Wright, Generalized means, Trans. Amer. Math. Soc. 98 (1961), 187-203.

4. C. Davis, Various averaging operations onto subalgebras, Illinois J. Math. 3 (1959), 538-553.

5. N. Dunford and J. T. Schwartz, Linear operators. I, Interscience, New York, 1958.

6. E. Michael, A linear transformation between function spaces, Notices Amer. Math. Soc. 8 (1961), 149.

Bell Telephone Laboratories, Murray Hill, New Jersey 